\title{
Fibra en los trastornos de la interacción intestino-cerebro
}

\section{Fiber in disorders of gut-brain interaction}

Karla R. García-Zermeño y José M. Remes-Troche*

${ }^{1}$ Laboratorio de Fisiología Digestiva y Motilidad Gastrointestinal, Instituto de Investigaciones Medico Biológicas, Universidad Veracruzana, Veracruz, Ver., México

\section{RESUMEN}

La suplementación con fibra dietaria (mediante el aumento de la ingesta de alimentos ricos en fibra o el uso de suplementos) es una intervención común que se ha utilizado para el manejo de trastornos metabólicos y gastrointestinales. En esta revisión narrativa, evaluamos el efecto terapéutico de la fibra en los trastornos de la interacción intestino-cerebro (TIIC), con especial atención en el estreñimiento crónico funcional (ECF) y el síndrome del intestino irritable (SII). En el caso del ECF la evidencia recomienda su uso, en especial la fibra soluble, ya que mejora el volumen de las heces y acelera el tránsito intestinal, y se emite con cautela la recomendación para la fibra insoluble por los efectos secundarios. En el caso del SII la evidencia es menor y mucho más heterogénea, y si bien mejora la consistencia y frecuencia de las evacuaciones, su efecto sobre el dolor abdominal es incierto. Por lo tanto, son necesarios más estudios de calidad para poder realizar recomendaciones específicas y conocer con claridad la dosis, tipo y fuente de fibra requerida para el manejo de los síntomas en ECF, SII y otros TIIC. Por otra parte, el consumo de alimentos naturales ricos en fibra y la coadministración de fibras (p. ej., psyllium más inulina) son opciones prometedoras que deben de explorarse.

Palabras clave: Fibra. Estreñimiento. Intestino irritable. Psyllium. Salvado.

\section{ABSTRACT}

Dietary fiber supplementation (through increasing the intake of high-fiber foods or using supplements) is a common intervention that has been used for the management of metabolic and gastrointestinal disorders. In this narrative review, we evaluate the therapeutic effect of dietary fiber in disorders of the gut-brain interaction (DGBI), with special attention to chronic idiopathic constipation (CIC) and irritable bowel syndrome (IBS). In the case of CIC, the evidence recommends its use, especially soluble fiber since it improves stool volume and accelerates intestinal transit, and the recommendation for insoluble fiber is issued with caution due to side effects. In the case of IBS, the evidence is less and much more heterogeneous, and although the consistency and frequency of bowel movements improves, its effect on abdominal pain is uncertain. Therefore, more high-quality studies are necessary to be able to make specific recommendations and clearly know the dose, and source of fiber required, for the management of symptoms in CIC, IBS and other DGBI. On the other hand, consumption of natural foods rich in fiber and the co-administration of fibers (for example, psyllium plus inulin) are promising options that should be explored.

Key words: Fiber. Constipation. Irritable bowel. Psyllium. Bran.

\author{
Correspondencia: \\ *José M. Remes Troche \\ E-mail: jose.remes.troche@gmail.com; joremes@uv.mx
}

NeuroGastroLatam Rev. 2021;5:154-169

Recibido: 15-05-2021

Aceptado: 11-08-2021

DOI: $10.24875 /$ NGL.21000010 


\section{INTRODUCCIÓN}

Los trastornos de la interacción intestino-cerebro (TIIC) se diagnostican y clasifican utilizando los criterios estandarizados recomendados por la Fundación Roma, en su IV versión ${ }^{1}$. En la actualidad, el tratamiento de estos trastornos se establece de acuerdo con su fisiopatología y el objetivo primario es lograr el control de los síntomas y mejorar la calidad de vida. Así pues, dentro de las opciones terapéuticas disponibles actualmente se incluyen cambios en el estilo de vida, modificaciones en la dieta y diversos fármacos que incluyen antiespasmódicos, laxantes, antidiarreicos, secretagogos y neuromoduladores. Una de las estrategias relacionadas con la dieta es la suplementación con fibra, la cual ha demostrado diversos beneficios para el mantenimiento de la salud y el tratamiento de múltiples condiciones, tanto gastrointestinales como no gastrointestinales.

Los estudios mecanísticos y los ensayos clínicos en fibras naturales o aisladas y extraídas han demostrado efectos reguladores en el intestino (p. ej., acelerar el tránsito orocecal, aumentar el peristaltismo, mejorar la consistencia de las heces por medio de la retención de líquido e incrementar el volumen de las heces) y efectos eubióticos (cambios en la composición de la microbiota intestinal y sus metabolitos) que tienen potenciales implicaciones terapéuticas para los TIIC. Las propiedades fisicoquímicas de diferentes fibras dietarias (como solubilidad, viscosidad y fermentabilidad) varían mucho según su origen y procesamiento, ya que determinan sus características funcionales y, por lo tanto, su utilidad clínica ${ }^{2}$. En esta revisión narrativa nos centraremos en discutir las características fisicoquímicas y funcionales de las diferentes fibras dietarias y el efecto terapéutico en los TIIC, con especial atención en el estreñimiento crónico funcional (ECF) y el síndrome del intestino irritable (SII), en donde existe más evidencia disponible.

\section{DEFINICIÓN Y CLASIFICACIÓN DE LA FIBRA}

Según la definición de la Organización Mundial de la Salud y el Codex Alimentarius ${ }^{3}$ (colección de normas, directrices y códigos de práctica reconocidos internacionalmente), la fibra dietaria incluye todos los carbohidratos que presentan un grado de polimerización de diez o más unidades monoméricas, que no son hidrolizados por las enzimas endógenas en el intestino delgado de los seres humanos, y que por lo tanto que pueden fermentarse de forma parcial o completa en el intestino grueso (principalmente ciego y colon derecho) ${ }^{4}$. El efecto de la fibra en el aparato digestivo depende de sus características fisicoquímicas, que incluyen: su estructura química, su fuente de origen, su solubilidad en agua, su viscosidad y su fermentabilidad (Fig. 1) .

\section{Características fisicoquímicas}

\section{SOLUBILIDAD}

La mayor parte de la evidencia actual se ha centrado en la solubilidad como una de las propiedades más importantes de la fibra debido al efecto que ejerce sobre la totalidad del tracto gastrointestinal. La solubilidad se refiere al grado en que las fibras pueden 


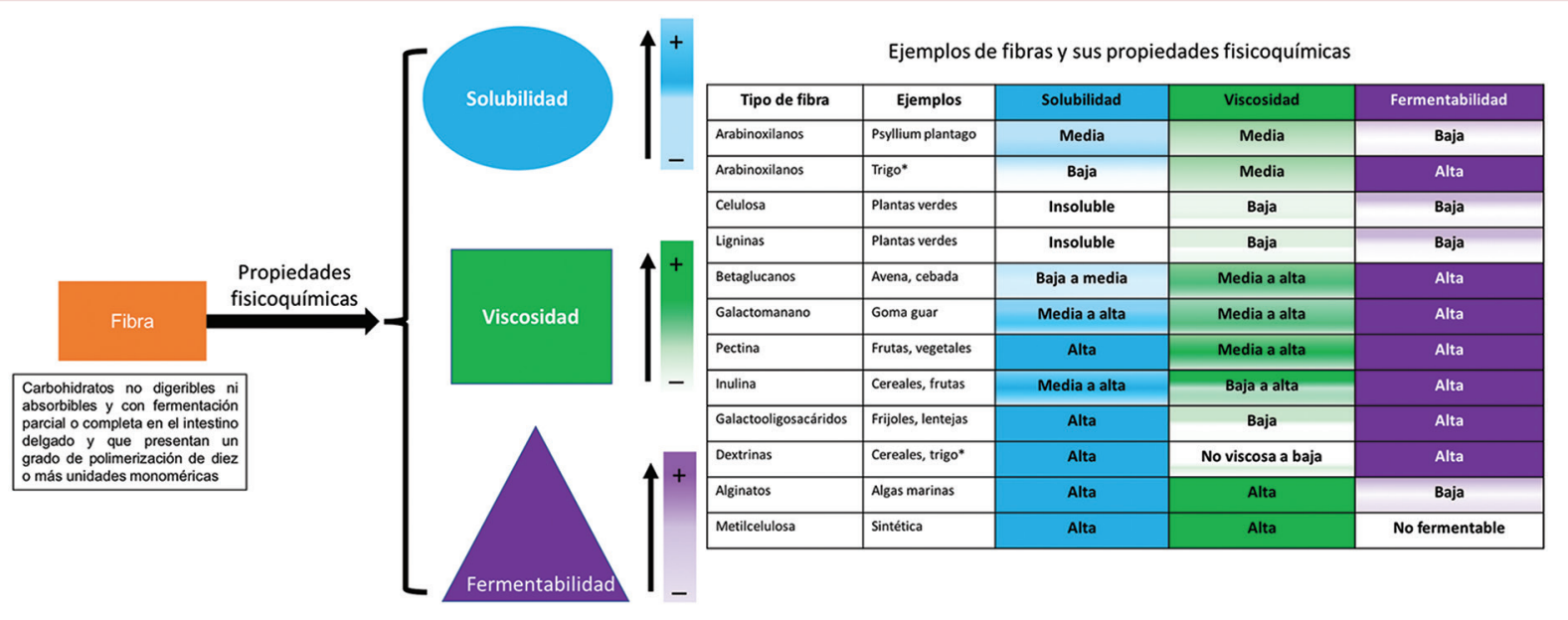

Figura 1. Propiedades fisicoquímicas de la fibra y ejemplos más comunes de estas. Se ilustra de acuerdo con la intensidad de los colores si esta propiedad es baja, media, alta o nula.

*El trigo contiene arabinoxilanos (polisacáridos heterogéneos no amiláceos) y dextrinas (oligosacáridos de poco peso molecular producidos por la hidrólisis del almidón), y ambos compuestos se clasifican como fibra.

disolverse en agua y con base en esto tradicionalmente se han clasificado como: solubles e insolubles ${ }^{2}$. A diferencia de las fibras insolubles, que permanecen como partículas discretas, las fibras solubles tienen una alta afinidad por el agua ${ }^{6}$. A esta característica se deben muchos de sus efectos fisiológicos en el intestino. Los primeros estudios in vitro de fibras aisladas permitieron distinguir entre las que afectan principalmente la absorción de glucosa y lípidos en el intestino delgado y las que afectan principalmente la función colónica, como el aumento de volumen de las heces y la disminución del tiempo de tránsito intestinal (como en el caso de la celulosa, el salvado de trigo y la lignina) $)^{7}$. Sin embargo, en 2003, la Organización de las Naciones Unidas para la Agricultura y la Alimentación propuso que estos términos relacionados con la solubilidad deberían eliminarse gradualmente debido a que su clasificación de solubilidad in vitro depende del método, y otras condiciones que pueden variar in vivo como el $\mathrm{pH}$ dentro del aparato digestivo, por lo que esta clasificación no necesariamente predice los efectos fisiológicos reales de la fibra ${ }^{8-10}$. Además, los alimentos enteros con alto contenido de fibra son a menudo una mezcla compleja de fibras solubles e insolubles (p. ej., almidón resistente, hemicelulosas, celulosa y lignina) y, por lo tanto, ejercen simultáneamente diferentes efectos fisiológicos en el tracto gastrointestinal. Por ejemplo, las manzanas contienen fracciones de fibra soluble (pectinas) e insoluble (celulosa) ${ }^{2}$. Incluso en modelos animales se ha demostrado que los efectos de las fibras solubles (es decir, distensión mecánica por la absorción de agua) e insolubles (aumento de volumen) en el íleon podrían activar el freno ileal (mecanismo de retroalimentación negativa que da como resultado la inhibición de la motilidad y secreción gastrointestinal) por medio 
mediadores como el péptido 1 similar al glucagón (GLP1) y el GLP2 ${ }^{11}$. No obstante, es importante destacar que aunque la solubilidad de forma aislada per se es un mal indicador de la función de la fibra, esta propiedad fisicoquímica junto con la viscosidad y la fermentabilidad son la causa de forma «global» de los efectos fisiológicas benéficos descritos en el tracto digestivo.

\section{VISCOSIDAD}

La viscosidad es el grado de resistencia al flujo. Generalmente se asocia con fibras solubles (p. ej., gomas, pectinas, $\beta$-glucanos y psyllium) y se relaciona con la capacidad, cuando está hidratada, de espesarse de una manera dependiente de la concentración ${ }^{12}$. Algunas formas de fibra, como las pectinas, tienen la capacidad de formar redes de gel ${ }^{13}$. El potencial de viscosidad de la fibra está determinado por algunas características como la longitud y estructura del polímero. Estos factores afectan el «tipo» de gel formado y la concentración crítica requerida para la formación de un gel viscoelástico. En la figura 1 se observa cuáles son las fibras con mayor y menor viscosidad. En términos generales, las fibras viscosas se pueden clasificar en dos grupos: polisacáridos en espiral aleatorios y polímeros de ensamblaje ordenados. Los polisacáridos en espiral aleatorios aumentan la viscosidad por medio del entrelazamiento, lo que restringe el flujo del solvente circundante. Los ejemplos incluyen los polímeros neutros como $\beta$-glucanos, psyllium, guar y galactomanano, en los que generalmente cuanto más largo es el polímero (es decir, mayor es el peso molecular), mayor es el entrelazamiento que se produce $y$, por lo tanto, menor es la concentración requerida para aumentar la viscosidad. Por el contrario, los polímeros de ensamblaje ordenados, como algunas pectinas y el alginato, forman una red de gel en presencia de iones divalentes (es decir, $\mathrm{Ca} 2+)^{14}$. El aumento de la viscosidad luminal intestinal tiene múltiples beneficios para la salud. Se ha demostrado que el consumo de fibra viscosa altera el tiempo de tránsito intestinal total, ya que disminuye el tiempo de vaciamiento gástrico y acelera el tiempo del tránsito del intestino delgado ${ }^{15}$.

Por otra parte, el aumento de la viscosidad luminal desempeña un papel fundamental en procesos metabólicos asociados a la función intestinal, ya que, por ejemplo, el retraso de la digestión disminuye la glucemia pospran$\operatorname{dia}^{16}$ y la lipemia ${ }^{17-19}$, y produce un aumento de la saciedad ${ }^{20}$. Además estudios in vitro han demostrado que fibras como el salvado de arroz y la celulosa interactúan con enzimas digestivas, inhibiendo la tasa de digestión de los nutrimentos ${ }^{21-23}$.

\section{FERMENTABILIDAD}

Cualquier carbohidrato no digerido que llegue al colon será fermentado (parcial o totalmente) por bacterias y en ese proceso se producen ácidos grasos de cadena corta (AGCC) y una serie de gases, incluidos el dióxido de carbono, el hidrógeno y el metano ${ }^{24,25}$. Los AGCC (principalmente acetato, propionato y butirato), a su vez, crean una carga osmótica, se absorben y se metabolizan posteriormente por los colonocitos, hepatocitos o tejidos periféricos ejerciendo múltiples efectos metabóli$\cos ^{26-28}$. La fermentación de la fibra también influye en el aumento de volumen fecal de manera indirecta, ya que la fermentación por 


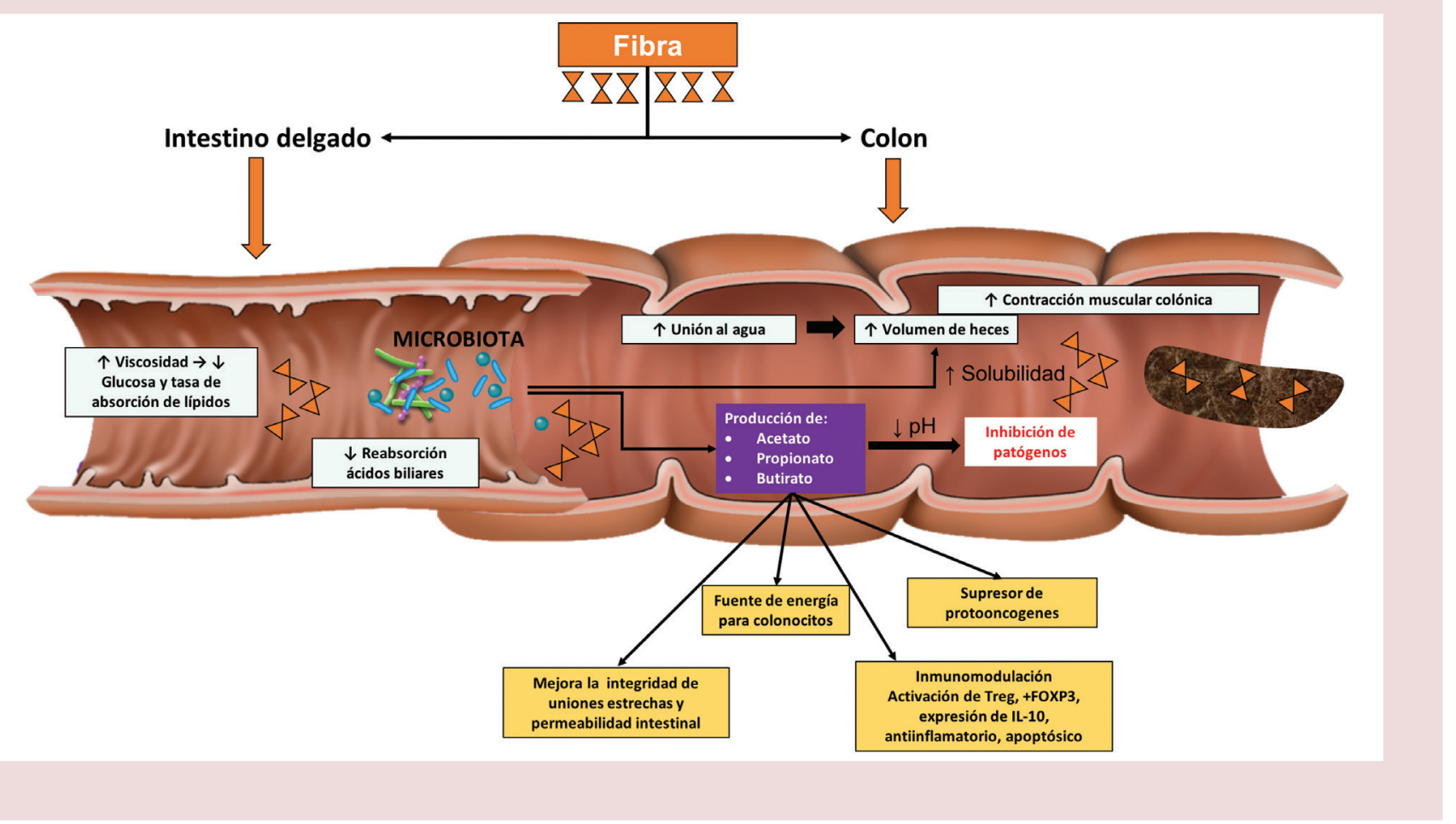

Figura 2. Mecanismos de acción de la fibra en intestino delgado y colon. En el intestino delgado la fibra produce un aumento de la viscosidad, incremento en la retención de agua y disminución de la reabsorción de sales miliares. Por otra parte, en el colon se incrementa más la retención de agua, lo que produce un mayor aumento en el volumen de las heces, lo que favorece la contracción colónica. La interacción con la microbiota produce múltiples efectos como inhibición de patógenos y producción de ácidos grasos de cadena corta que ejercen múltiples efectos metabólicos e inmunomoduladores.

Treg: células T reguladoras; FOXP3: proteína P3 de la forkhead box, IL: interleucina.

la microbiota del colon estimula el crecimiento $\mathrm{y}$ aumento de la biomasa microbiana ${ }^{29}$. También influye en el aumento de volumen fecal directamente por medio de la retención de agua (Fig. 2) ${ }^{30-32}$. Sin embargo, el efecto secundario no deseado de la ingestión de fibra y la fermentación resultante es la producción de gas. Este gas a menudo es maloliente $\mathrm{y}$, a su vez, puede causar dolor, distensión y flatulencias. Esta característica de algunas fibras puede ser particularmente relevante para los pacientes con TIIC, en especial aquellos que más padecen distensión abdominal. En la figura 1 se destaca cómo dentro de las fibras que más se fermentan están la inulina, los galactooligosacáridos y los alginatos, mientras que la metilcelulosa y las ligninas son poco fermentables.

\section{MECANISMO DE ACCIÓN DE LA FIBRA DIETARIA}

\section{Efectos sobre la función gastrointestinal}

Las propiedades de la fibra dietaria para mejorar la función intestinal han sido descritas desde principios de la década de $1970^{33}$. En los estudios pioneros de Burkitt y Tomlin se 
determinó que la fibra insoluble (p. ej., salvado de trigo) aumenta la masa fecal y la tasa de tránsito colónico por medio de la estimulación/irritación mecánica de la mucosa intestinal, induciendo secreción y peristaltismo ${ }^{34}$. Posteriormente se demostró que tanto el tamaño como la forma de las partículas de fibra (propiedades fisicoquímicas) eran determinantes, ya que las partículas grandes y gruesas proporcionaban una mayor eficacia laxante que las partículas finas y suaves ${ }^{35}$, y que además de ejercer un efecto mecánico (el aumento del volumen de las heces) podría ejercer efectos metabólicos locales como al incremento de la biomasa y los subproductos de la fermentación, como el gas y los $\mathrm{AGCC}^{36}$.

En la actualidad se reconoce que el potencial efecto terapéutico de la fibra está relacionado con múltiples mecanismos que incluyen: la aceleración del tránsito oroanal, la disminución de la presión intracolonica ${ }^{37,38}$, y mediante sus efectos relacionados con la microbiota, la disminución de la inflamación de bajo grado y una menor permeabilidad intestinal (Fig. 2) ${ }^{39}$. Sin embargo, el elevado consumo de fibra (por ejemplo 30 gramos de psyllium al día) también puede retrasar el tránsito de gas, al disminuir la propulsión del bolo al recto $^{40}$ y en el caso de la fibra altamente fermentable (galactooligosacáridos) promover una mayor producción de gas.

La fibra soluble, viscosa y mínimamente fermentable tiene una alta capacidad de retención de agua y formación de gel que se conserva en todo el colon, normalizando la forma de las heces ${ }^{41,42}$. La consistencia de las heces tiene una alta correlación con el contenido de agua en las heces, y un cambio relativamente pequeño en el contenido de agua en las heces (aumento del 4.7\%) puede dar lugar a un efecto de ablandamiento (diferencia de viscosidad de 4.6 veces) ${ }^{41}$.

Hay que mencionar también que la fibra también tiene efectos extracolónicos principalmente a nivel gástrico ${ }^{20,43-46}$. En general, las dosis altas ( $\geq 7 \mathrm{~g}$ ) de salvado de trigo, inulina y $p s y$ llium tienden a retrasar el vaciamiento gástrico, mientras que las dosis más bajas no muestran un efecto significativo. El retraso en el vaciamiento gástrico puede deberse a una mayor viscosidad del contenido gástrico, lo que reduce el flujo pilórico. El aumento de la viscosidad reduce la capacidad de dilución de los sólidos $\mathrm{y}$, por lo tanto, el vaciamiento gástrico se ve puede retardar ${ }^{47,48}$. También es posible que a esas dosis la fibra disminuya la absorción de nutrimentos en el intestino delgado, lo que puede a su vez afectar el vaciamiento gástrico que normalmente ocurre cuando los nutrimentos ingresan al duodeno ${ }^{49}$. Esto podría explicar los síntomas de distensión y sensación de distensión abdominal provocados por el salvado $^{50}$. Así pues, parece ser que evitar el uso de dosis altas de fibra tiene un efecto conciliador sobre la función gastrointestinal, disminuyendo la posibilidad de retraso en el vaciamiento gástrico y la retención de gas, pero promoviendo un mejor tránsito intestinal.

\section{Efectos sobre la microbiota}

Existe evidencia de que los cambios en el complejo ambiente gastrointestinal por la fibra influyen de forma positiva en la microbiota fecal, quizás debido a la producción variada de AGCC con altas concentraciones luminales resultantes de la fermentación por la reducción del pH en colón (5.5 a 6.5 en el 
colon proximal, donde la fermentación es mayor, en comparación con un pH de 6.5 a 7.0 en el colon distal) e inhiben el crecimiento de enterobacterias gramnegativas, incluidos los patógenos familiares a Salmonella spp. y Escherichia coli, y promoviendo el crecimiento de bacterias benéficas ${ }^{51,52}$.

Los oligosacáridos (moléculas constituidas por la unión de 2 a 9 monosacáridos cíclicos) como la inulina y otras fibras solubles se fermentan en el colon proximal por bacterias endógenas produciendo AGCC, que a su vez ejercen efectos moduladores sobre la microbiota intestinal. Por lo tanto, algunas fibras fermentables pueden ejercer un efecto prebiótico, y por lo tanto clasificarse como tal, ya que sirven como "sustrato que es utilizado selectivamente por los microorganismos hospedadores que confieren un beneficio para la salud» ${ }^{53}$. Algunos estudios han mostrado que una ingesta habitualmente alta de fibra dietaria genera una mayor respuesta microbiana intestinal a los prebióticos (es decir, aumento en la abundancia de Bifidobacterium y Faecalibacterium, y disminución en Coprococcus, Dorea y Ruminococcus) en comparación con aquellos con una ingesta de fibra dietaria habitualmente baja, lo que sugiere que las personas que siguen una dieta habitual alta en fibra tienen más probabilidades de beneficiarse de una fibra con actividades prebióticas como la inulina ${ }^{53}$.

\section{FUENTES DE OBTENCIÓN DE LA FIBRA DIETARIA}

Habitualmente la fibra debe consumirse como parte de la dieta en forma de vegetales y frutas. Aunque se recomienda que el consumo diario promedio en adultos debe ser entre 25 y 30 g de fibra, en promedio la población consume de 10 a $18 \mathrm{~g}^{54}$. Por ejemplo, el consumo diario de fibra en México es inferior a la cantidad recomendada y está relacionada con la disponibilidad de la fibra en la dieta. De acuerdo con la revisión de Barquera, et al. ${ }^{55}$, las mujeres de comunidades rurales consumen en promedio $23.6 \mathrm{~g}$ al día; es decir, consumen más fibra que el promedio nacional (17.3 g), y aún más si se compara con el medio urbano (15.5 g).

En el manejo de los TIIC y con fines de suplementar, se han utilizado fibras extraídas de alimentos naturales o fibras sintéticas (metilcelulosa), cuyas características fisicoquímicas y bioaccesibilidad pueden ser diversas ${ }^{2}$. Recientemente se ha insistido en tratar de utilizar fuentes naturales de fibra dietaria, ya que a menudo estas pueden contener una mayor concentración de nutrimentos y compuestos bioactivos (como los polifenoles), y diferentes tipos de fibra a la vez (p. ej., el trigo contiene arabinoxilanos y dextrinas). Sin embargo, en el procesamiento de las fibras a partir de su forma natural sus propiedades funcionales y la concentración de sus nutrimentos pueden cambiar. Ejemplos de estas combinaciones naturales de fibras incluyen a la tuna, el galactomanano, la cáscara de plátano, la calabaza, la raíz de yacón, Moringa oleifera, el nopal, el agave, el kiwi y la ciruela pasa. No obstante, si bien existen estudios clínicos al respecto, es importante mencionar que la evidencia es aún limitada.

\section{EVIDENCIA CLÍNICA DEL USO DE FIBRA DIETARIA EN ESTREÑIMIENTO}

Una dieta alta en fibra es a menudo la primera recomendación en el tratamiento de un 
paciente con $\mathrm{ECF}^{56,57}$. A pesar de la heterogeneidad inherente de los datos en muchos de los estudios en pacientes con ECF, parece haber una mejoría significativa en los síntomas de estreñimiento y molestias abdominales comparadas con placebo con la fibra soluble (psyllium, inulina), por lo que muchos consensos (Tabla 1) recomiendan el uso de fibra ${ }^{58-69}$. Por ejemplo, en el consenso Mexicano para el estreñimiento ${ }^{58}$, en pacientes con una ingesta baja de fibra se recomienda el consumo de alimentos con alto contenido o suplementos, ya que estos pueden aumentar la frecuencia de las evacuaciones (calidad de la evidencia y fuerza de la recomendación: alimentos ricos en fibra = débil a favor de la intervención; suplementos de fibra = fuerte a favor de la intervención; ingesta de líquidos = débil a favor de la intervención). Además, se insiste en que la ingesta de líquidos (1.5 a 2 litros por día) puede mejorar el estreñimiento y potenciar los efectos de la fibra en la dieta y en los suplementos.

Muy pocos estudios han evaluado el consumo de alimentos ricos en fibra para el tratamiento del estreñimiento. En un estudio con 40 pacientes con ECF que fueron asignados aleatoriamente a recibir $50 \mathrm{~g}$ de ciruelas dos veces al día u $11 \mathrm{~g}$ de psyllium dos veces al día por tres semanas, se demostró que el consumo de ciruelas aumentó el número de evacuaciones espontáneas completas (EEC) $(3.5 \pm$ 0.2 vs. $2.8 \pm 0.2 ; \mathrm{p}=0.006)$ y disminuyó la consistencia de las evacuaciones (3.2 vs. 2.8; $\mathrm{p}=0.02)^{70}$. En otro estudio con 33 pacientes $\mathrm{y}$ 20 controles sanos se demostró que el consumo de dos kiwis al día durante cuatro semanas mejoró el número de EEC comparado con su basal ( $2.2 \pm 2.6$ vs. $4.4 \pm 4.6 ; \mathrm{p}=0.013)$, los síntomas de estreñimiento $(\mathrm{p}=0.02)$ y aceleró el tránsito intestinal $(\mathrm{p}=0.003)^{71}$.
En un estudio publicado recientemente Chey, et al. ${ }^{72}$ asignaron de forma aleatoria a 79 pacientes con ECF a recibir dos kiwis verdes o 100 gramos de ciruelas pasas o 12 gramos de psyllium al día durante cuatro semanas. La respuesta global (de acuerdo con el número de EEC) fue del $46 \%$ para el grupo de kiwis, el $67 \%$ para el grupo que recibió ciruelas y el 64\% para el Psyllium ( $\mathrm{p}=0.22$ ). También se demostró que hubo un aumento significativo en el número de EEC con los tres tratamientos ( $\mathrm{p} \leq 0.003) ;$ la consistencia de las heces mejoró significativamente con kiwis $(\mathrm{p}=0.01)$ y ciruelas pasas $(\mathrm{p}=0.049)$; y el esfuerzo mejoró significativamente con kiwis $(p=0.003)$, ciruelas pasas $(\mathrm{p}<0.001)$ y psyllium $(\mathrm{p}=0.04)$. Los pacientes asignados al grupo de kiwis informaron una mejora significativa en las puntuaciones de distensión $(\mathrm{p}=0.02)$.

Por otra parte, un ensayo clínico controlado doble ciego llevado a cabo por Coss-Adame, et al. ${ }^{73}$ evalúo la eficacia de fructanos de agave (a dosis de 5, 10 o $5 \mathrm{~g}$ en combinación con $10 \mathrm{~g}$ de maltrodextrinas) vs. $15 \mathrm{~g}$ de Plantago psyllium en pacientes con ECF (Roma III). Los resultados reportados fueron el número de evacuaciones completas, que aumentó en todos los brazos, y todos los grupos reportaron mejoría en los síntomas (dolor abdominal, pujo, tenesmo, distensión y flatulencias).

Respecto a los suplementos de fibra, varios metaanálisis han evaluado sus efectos benéficos ${ }^{74,75}$. En el metaanálisis realizado por Suares, et al. ${ }^{76}$, donde se incluyeron seis estudios, es clara la heterogeneidad de estos: las cantidades de fibra oscilaron entre 10 y $20 \mathrm{~g}$ de fibra/día, la duración de tratamiento de dos a ocho semanas y cuatro de los ensayos elegibles utilizaron fibra soluble (tres con psyllium, 
TABLA 1. Recomendaciones respecto al uso de la fibra en síndrome del intestino irritable (SII) y estreñimiento crónico (EC) emitidas por guías y/o consensos de las asociaciones de gastroenterología más importantes

\begin{tabular}{|c|c|c|c|c|}
\hline & $\begin{array}{l}\text { Síndrome de intestino } \\
\text { irritable }\end{array}$ & $\begin{array}{l}\text { Recomendación/ } \\
\text { calidad de la } \\
\text { evidencia }\end{array}$ & Estreñimiento crónico & $\begin{array}{l}\text { Recomendación/ } \\
\text { calidad de la } \\
\text { evidencia }\end{array}$ \\
\hline AMG 58,59 & $\begin{array}{l}\text { La ingestión de fibra soluble } \\
\text { es benéfica en el SII } \\
\text { La ingestión de salvado no } \\
\text { mejora los síntomas del SI }\end{array}$ & $\begin{array}{l}\text { Fibra soluble: fuerte/ } \\
\text { alta } \\
\text { Salvado: débil/baja }\end{array}$ & $\begin{array}{l}\text { En pacientes con una ingesta baja de } \\
\text { fibra se recomienda el consumo de } \\
\text { alimentos con alto contenido o suplemen- } \\
\text { tos, ya que estos pueden aumentar la } \\
\text { frecuencia de las evacuaciones } \\
\text { La ingesta de líquidos ( } 1.5 \text { a } 2 \text { I por día) } \\
\text { puede mejorar el estreñimiento y } \\
\text { potenciar los efectos de la fibra en la } \\
\text { dieta y de los suplementos }\end{array}$ & $\begin{array}{l}\text { Alimentos ricos en fibra: } \\
\text { débil/baja } \\
\text { Suplementos de fibra: } \\
\text { fuerte/moderada }\end{array}$ \\
\hline $\mathrm{BSG}^{60}$ & $\begin{array}{l}\text { La fibra soluble, como la } \\
\text { ispaghula, es un tratamiento } \\
\text { eficaz para los síntomas } \\
\text { generales y el dolor } \\
\text { abdominal en el SII, pero } \\
\text { debe evitarse la fibra } \\
\text { insoluble (p. ej., salvado de } \\
\text { trigo), ya que puede } \\
\text { exacerbar los síntomas } \\
\text { La fibra soluble debe } \\
\text { iniciarse en una dosis baja } \\
\text { (3-4 g/día) y aumentarse } \\
\text { gradualmente para evitar la } \\
\text { distensión }\end{array}$ & Fuerte/moderada & No existen guías al respecto & - \\
\hline ESNM $^{61}$ & No existen guías al respecto & - & $\begin{array}{l}\text { El uso de fibra soluble (psyllium) es } \\
\text { efectiva para el manejo del estreñimiento } \\
\text { La utilidad de los agentes formadores de } \\
\text { bolo, en particular fibras insolubles. esta } \\
\text { limitada por eventos adversos, particular- } \\
\text { mente distensión, flatulencia y dolor } \\
\text { abdominal }\end{array}$ & Fuerte/moderada \\
\hline $\mathrm{ACG}^{62}$ & $\begin{array}{l}\text { Se sugiere que se utilice } \\
\text { fibra soluble, pero no } \\
\text { insoluble, para tratar los } \\
\text { síntomas globales del SII }\end{array}$ & Fuerte/moderada & No existen guías al respecto & - \\
\hline $\mathrm{AGA}^{63}$ & $\begin{array}{l}\text { No existen recomendaciones } \\
\text { al respecto }\end{array}$ & - & $\begin{array}{l}\text { Se sugiere un aumento gradual de la } \\
\text { ingesta de fibra en especial soluble } \\
\text { (psyllium } 15 \mathrm{~g} \text { ) }\end{array}$ & - \\
\hline SEPD ${ }^{64}$ & $\begin{array}{l}\text { Una dieta con alimentos de } \\
\text { alto contenido en fibra } \\
\text { soluble (como las ciruelas } \\
\text { secas) ha demostrado } \\
\text { beneficio en la mejoría del } \\
\text { estreñimiento leve, pero no } \\
\text { en la del dolor o la } \\
\text { distensión abdominal, } \\
\text { pudiendo incluso empeorar } \\
\text { estos síntomas en pacientes } \\
\text { con SII }\end{array}$ & $\begin{array}{l}\text { No califican la } \\
\text { evidencia }\end{array}$ & $\begin{array}{l}\text { La utilización de fibra dietaria o suplemento } \\
\text { de fibra como primera medida terapéutica } \\
\text { es razonable en cualquier paciente con } \\
\text { estreñimiento, asocie o no molestias } \\
\text { abdominales, existiendo mayor evidencia } \\
\text { con la fibra soluble, pudiendo considerarse } \\
\text { que un curso de tratamiento de } 6 \text { semanas } \\
\text { es suficiente para evaluar su eficacia } \\
\text { Se debe prestar atención no solo a la } \\
\text { eficacia, sino también a la tolerancia, por } \\
\text { lo que es aconsejable ir aumentando la } \\
\text { cantidad de fibra de forma progresiva }\end{array}$ & No califican la evidencia \\
\hline
\end{tabular}


TABLA 1. Recomendaciones respecto al uso de la fibra en síndrome del intestino irritable (SII) y estreñimiento crónico (EC) emitidas por guías y/o consensos de las asociaciones de gastroenterología más importantes (Continuación)

\begin{tabular}{|c|c|c|c|c|}
\hline & $\begin{array}{l}\text { Síndrome de intestino } \\
\text { irritable }\end{array}$ & $\begin{array}{l}\text { Recomendación/ } \\
\text { calidad de la } \\
\text { evidencia }\end{array}$ & Estreñimiento crónico & $\begin{array}{l}\text { Recomendación/ } \\
\text { calidad de la } \\
\text { evidencia }\end{array}$ \\
\hline WGO $0^{65,66}$ & $\begin{array}{l}\text { Una dieta rica en fibras o } \\
\text { formadora de volumen (p. ej., } \\
\text { psyllium) combinado con una } \\
\text { ingesta suficiente de líquidos } \\
\text { parecería ser un enfoque } \\
\text { lógico en SII } \\
\text { Las fibras insolubles pueden } \\
\text { exacerbar los síntomas y dar } \\
\text { poco alivio. Pueden incluso } \\
\text { provocar eventos adversos e } \\
\text { inflamación, distensión, } \\
\text { flatulencia y dolor abdominal, } \\
\text { que pueden limitar el uso de } \\
\text { la fibra insoluble, especial- } \\
\text { mente si los aumentos de la } \\
\text { ingesta de fibras no se } \\
\text { introducen gradualmente. } \\
\text { Por otro lado, las fibras } \\
\text { solubles como el psyllium } \\
\text { (ispaghula) alivian el SII }\end{array}$ & $\begin{array}{l}\text { No se califica la } \\
\text { evidencia }\end{array}$ & $\begin{array}{l}\text { La modificación de la dieta puede } \\
\text { consistir en agregar fibras para lograr una } \\
\text { dieta rica en fibras ( } 25 \mathrm{~g} \text { de fibras) y } \\
\text { suplementos de líquidos (hasta } 1.5-2.0 \mathrm{l} \\
\text { día); puede mejorar la frecuencia de las } \\
\text { evacuaciones y disminuir la necesidad de } \\
\text { laxantes }\end{array}$ & $\begin{array}{l}\text { No se califica la } \\
\text { evidencia }\end{array}$ \\
\hline NICE ${ }^{67,68}$ & $\begin{array}{l}\text { Los profesionales de la salud } \\
\text { deben revisar la ingesta de } \\
\text { fibra de las personas con SII, } \\
\text { ajustándola (generalmente } \\
\text { reduciéndola) mientras } \\
\text { monitorean el efecto sobre } \\
\text { los síntomas } \\
\text { Se debe disuadir a las } \\
\text { personas con SII de } \\
\text { consumir fibra insoluble (p. } \\
\text { ej., salvado). Si se recomien- } \\
\text { da un aumento de fibra } \\
\text { dietaria, debe ser fibra } \\
\text { soluble como polvo de } \\
\text { ispaghula o alimentos con } \\
\text { alto contenido de fibra } \\
\text { soluble (p. ej., avena) }\end{array}$ & $\begin{array}{l}\text { No se califica la } \\
\text { evidencia }\end{array}$ & $\begin{array}{l}\text { Se recomienda incluir alimentos con alto } \\
\text { contenido de fibra (como frutas, verduras, } \\
\text { pan con alto contenido de fibra, frijoles } \\
\text { horneados y cereales integrales para el } \\
\text { desayuno) (no se aplica a los lactantes } \\
\text { exclusivamente amamantados) } \\
\text { No recomiende el salvado sin procesar, } \\
\text { que puede causar hinchazón y flatulencia } \\
\text { y reducir la absorción de micronutrimen- } \\
\text { tos }\end{array}$ & $\begin{array}{l}\text { No se califica la } \\
\text { evidencia }\end{array}$ \\
\hline $\begin{array}{l}\text { Consenso } \\
\text { latinoameri- } \\
\text { cano }^{69}\end{array}$ & No existen guías al respecto & - & $\begin{array}{l}\text { Las evidencias sugieren que pueden ser } \\
\text { de utilidad en algunos pacientes el } \\
\text { psyllium el policarbófilo y la } \\
\text { metilcelulosa }\end{array}$ & $\begin{array}{l}\text { psyllium: } \\
\text { fuerte/moderada } \\
\text { Policarbófilo: débil/baja }\end{array}$ \\
\hline
\end{tabular}

SII: síndrome de intestino irritable; AMG: Asociación Mexicana de Gastroenterología; BSG: Sociedad Británica de Gastroenterología; ESNM: Sociedad Europea de Neurogastroenterología y Motilidad; ACG: Colegio Americano de Gastroenterología; AGA: Asociación Americana de Gastroenterología; SEPD: Sociedad Española de Patología Digestiva; WGO: Organización Mundial de Gastroenterología; NICE: Instituto Nacional de Excelencia en Salud y Atención.

uno con inulina y maltodextrina) y dos estudios utilizaron fibra insoluble (salvado de trigo en un estudio y pan de centeno en el otro) ${ }^{77-82}$. El ensayo más grande fue un ensayo clínico aleatorizado simple ciego con 201 pacientes de atención primaria que sometieron a tratamiento por un periodo de dos semanas $^{77}$. El $87 \%$ de los pacientes asignados al 
psyllium informaron una mejoría en los síntomas, en comparación con el $47 \%$ de los pacientes que recibieron placebo ( $p<0.001$ ). También hubo una respuesta significativa en cuanto al dolor/malestar abdominal y el esfuerzo al defecar. Se observaron efectos similares entre los otros tres ensayos de fibra soluble. En otro estudio, el dolor con la defecación se redujo significativamente con psyllium, pero el 18\% de los pacientes con psyllium informaron dolor abdominal como un efecto secundario en comparación con el $0 \%$ del pla$\mathrm{cebo}^{78}$. En el estudio donde se evalúo el salvado de trigo ${ }^{81}$, en los 24 pacientes reclutados para recibir $20 \mathrm{~g}$ de salvado por día o placebo no hubo una diferencia estadísticamente significativa en la respuesta. Para el estudio del pan de centeno ${ }^{82}, 29$ mujeres consumieron pan de centeno (37 g/día de fibra) o pan bajo en fibra (6.6 g/día de fibra) durante un periodo de tres semanas. Después del periodo de intervención, la diferencia media en el número de evacuaciones por día fue 0.3 más alta para los pacientes asignados al pan de centeno en comparación con los que recibieron pan con bajo contenido de fibra ( $p=0.001$ ). La dificultad para defecar también se redujo significativamente con pan de centeno $(p<0.001)$ $\mathrm{y}$ las heces fueron más blandas ( $\mathrm{p}<0.001)$. Sin embargo, hubo puntuaciones de síntomas más altas para los efectos secundarios gastrointestinales como dolor abdominal, flatulencia, borborigmos y distensión con el pan de centeno en comparación con el pan con bajo contenido de fibra.

Otro metaanálisis realizado en 2016 que evaluó siete ensayos clínicos controlados demostró que el 77\% de los que recibieron suplementos de fibra respondieron, en comparación con el $44 \%$ de los que recibieron placebo (riesgo relativo [RR] de éxito: 1.71; intervalo de confianza del 95\% [IC 95\%]: 1.20-2.42; $\mathrm{p}=0.003)$ y se estima un número necesario que tratar de 3 (IC 95\%: 2.6-3.4) ${ }^{83}$. La fibra incrementó la frecuencia de las evacuaciones ( $p=0.03$ ) y disminuyó la consistencia de las heces $(p=0.02)$. En 2019 , de Vries, et al. ${ }^{84}$ realizaron una revisión sistemática acerca del efecto de los $\beta$-fructanos (un ejemplo de fibras dietarias solubles que están presentes de forma natural en diversas frutas y verduras incluida la achicoria) sobre la función intestinal incluyendo SII y ECF. Se seleccionaron 47 publicaciones para su inclusión. El parámetro principal fue la frecuencia de las deposiciones, evaluada por el número de defecaciones por día durante el periodo de estudio. Los resultados secundarios fueron la consistencia de las heces, el peso de las heces secas y húmedas y el tiempo de tránsito. Los autores demuestran que los $\beta$-fructanos de cadena corta contribuyeron a aumentar la frecuencia de las deposiciones ( 0.36 defecación \pm 0.06 por día; $\mathrm{p}<0.001)$, mientras que no se informó ningún efecto significativo con los $\beta$-fructanos de cadena larga $(-0.03 \pm 0.11 ; \mathrm{p}=0.82)$. También se demostró estadísticamente un aumento mínimo en el peso de las heces con $\beta$-fructanos de cadena corta.

Aproximadamente la mitad de los pacientes con síntomas refractarios al tratamiento convencional de estreñimiento tienen un tiempo de tránsito intestinal prolongado y hasta un $15 \%$ disinergia ${ }^{85}$. Hay pocos estudios que hayan evaluado la eficacia de la fibra para el estreñimiento en otras formas de EC, como con el EC con tránsito lento o la defecación disinérgica. Un estudio no aleatorizado demostró que el 88\% de los pacientes con tránsito lento y el $63 \%$ de los pacientes con un 
trastorno de la defecación no respondieron al tratamiento con $30 \mathrm{~g}$ de fibra por día, mientras que el $85 \%$ de los pacientes con EC sin estos hallazgos mejoraron o quedaron libres de síntomas ${ }^{86}$.

En resumen, la fibra más estudiada en ECF es Plantago psyllium, una fibra fermentable con solubilidad intermedia ${ }^{87}$. La evidencia para recomendar goma guar, pectina, metilcelulosa y policarbófilo es limitada y se necesitan más estudios para evaluar su eficacia en $\mathrm{ECF}^{88}$. La evidencia sugiere que el efecto terapéutico de la fibra es mayor cuando se consumen más de $15 \mathrm{~g}$ al día. Sin embargo, es muy importante mencionar que en algunos pacientes la fibra dietaria o los suplementos pueden agravar los síntomas asociados al estreñimiento como distensión abdominal y flatulencias, por lo que la dosis recomendada va a depender de la tolerancia de cada paciente.

La escasez de datos de alta calidad destaca la necesidad de realizar más ensayos clínicos controlados con metodología rigurosa y que utilicen como objetivos primarios aquellos que más se apeguen a las definiciones de Roma IV ${ }^{89}$.

\section{EVIDENCIA CLÍNICA DEL USO DE FIBRA DIETARIA EN SÍNDROME DE INTESTINO IRRITABLE}

Históricamente, el aumento de la ingesta de fibra ha sido una recomendación estándar para los pacientes con SII. Sin embargo, aunque el uso de la fibra para el SII sigue siendo controvertido, las pautas para el consumo de fibra en sujetos con SII varían (Tabla 1). El National Institute for Health and Care
Excellence $^{67}$ en el Reino Unido recomienda que se reduzca la ingesta de almidón resistente, mientras que las Pautas Globales de la Organización Mundial de Gastroenterología ${ }^{65}$ sugieren que deben fomentarse los alimentos ricos en fibra o los suplementos de fibra ( $p$. ej., psyllium) y que el consumo de fibras insolubles que pueden exacerbar los síntomas debe limitarse. En el consenso mexicano para el manejo del SII se considera que la evidencia para la fibra soluble tiene una calificación de A2 fuerte a favor de la intervención de acuerdo con la clasificación de GRADE (Grading of Recommendations, Assessment, Development and Evaluation), mientras que la evidencia para el salvado es B2 débil en contra de la intervención ${ }^{68}$.

En uno de los primeros estudios sobre fibra y SII, en 1977, Manning, et al..$^{90}$ examinaron el efecto de una dieta alta o baja en fibra durante seis semanas sobre el dolor abdominal y la frecuencia de las evacuaciones en 26 pacientes. Los participantes en este ensayo simple ciego ingirieron $20 \mathrm{~g}$ adicionales de salvado de trigo por día. Los investigadores encontraron una mejora significativa en la frecuencia $(\mathrm{p}<0.05)$ y la gravedad del dolor $(\mathrm{p} \sim 0.01)$. El hábito intestinal mejoró en el grupo con alto contenido de fibra $(\mathrm{p}<0.05)$ y la frecuencia intestinal también mejoró modestamente $(\mathrm{p}<0.02)$. Otro ensayo posterior que utilizó psyllium en 80 pacientes con SII demostró que este tipo de fibra mejoró significativamente los síntomas de estreñimiento $(p=0.026)$ y el tiempo de tránsito $(p=0.001)$, pero no la distensión ni el dolor abdominal ${ }^{91}$. Si bien uno pudiera pensar que la fibra solo es útil en el SII con estreñimiento, un estudio con 72 pacientes (independientemente del subtipo) demostró que una dieta con «alto contenido 
de fibra» (30 g de fibra/día) mejoró la consistencia de las heces, la frecuencia intestinal y la urgencia, y no produjo ningún cambio en la distensión abdominal o las flatulencias ${ }^{92}$. Así pues, este estudio sugiere que los síntomas que más se benefician de la prescripción de una dieta alta en fibra son las heces duras, el estreñimiento y la urgencia.

Hasta la fecha, varias revisiones sistemáticas y metaanálisis han concluido que algunas fibras son benéficas para reducir los síntomas del SII y mejorar la frecuencia y consistencia de las heces, aunque los resultados son inconsistentes, con una amplia variación en las respuestas $^{93-96}$. Los beneficios parecen limitarse a la fibra soluble (RR de tener síntomas continuos después de la suplementación de 0.83 (IC 95\%: 0.73-0.94) en comparación con otras fibras como el salvado (RR: 0.90; IC 95\%: 0.79-1.03) ${ }^{96}$.

Recientemente, Remes-Troche, et al..$^{97}$ publicaron los resultados de un ensayo clínico controlado, paralelo, doble ciego, para evaluar el efecto de una fibra de nopal (Opuntia ficusindica) durante una semana en pacientes con SII de acuerdo con Roma IV. Es importante mencionar que el nopal es un cultivo nativo de México (25 de 32 Estados cultivan el cactus), que, a pesar de la limitada revisión científica, se ha consumido por sus beneficios de promoción de la salud durante miles de años. Se ha demostrado que estos tallos tienen un índice glucémico bajo y son ricos en fibra, especialmente en su forma deshidratada (polvo), por lo que se ha utilizado para el manejo del síndrome metabólico ${ }^{98}$. En este estudio 60 pacientes fueron asignados aleatoriamente $\mathrm{y}$ a ciegas para recibir fibra de nopal (10, 20 o 30 g/día) o placebo (30 g/día de dextrosa) durante una semana. La fibra utilizada contenía por cada 100 g: 37.6\% de fibra insoluble, $13.2 \%$ de fibra soluble, $<2 \%$ de almidón, $4.69 \%$ de fructosa, $3.62 \%$ de glucosa y $1.81 \%$ de fructanos. La gran mayoría de los pacientes reportaron alivio adecuado de los síntomas después de 20 g/día (87\%; p = 0.008) y $30 \mathrm{~g} /$ día (80\%; $\mathrm{p}=0.025)$ de fibra de nopal en comparación con el placebo (33\%). Los pacientes que recibieron $20 \mathrm{~g} /$ día de fibra de nopal (67\%) tuvieron una reducción $>50 \%$ en la gravedad de los síntomas en comparación con placebo (20\%; p = 0.027), mientras que la dosis de $30 \mathrm{~g}$ /día indujo más evacuaciones blandas ( $p=0.027)$. Las tasas de respuesta fueron similares entre los subtipos de SII. Si bien los resultados son prometedores, es importante destacar que es un ensayo de muy corta duración con el fin de cumplir la «prueba de concepto», por lo que es necesario evaluar si los efectos reportados en este estudio son similares a largo plazo.

En resumen, aunque existen pocos estudios metodológicamente rigurosos y con la potencia adecuada que hayan examinado el papel de las fibras disponibles para el tratamiento de los síntomas del SII, los resultados sugieren que la suplementación con fibras tanto insolubles como solubles pueden ser útil en algunos pacientes. Sin embargo, se requieren más ensayos con una metodología más estricta, además de que existe una necesidad urgente de evaluar los efectos de la fibra en los subgrupos del SII para permitir una mejor comprensión de su potencial terapéutico.

\section{CONCLUSIÓN}

La fibra dietaria, por sus características fisiológicas y múltiples efectos a nivel del 
tránsito digestivo por medio de la modulación de la motilidad gástrica y del intestino delgado, la absorción intestinal, la microbiota colónica y la fermentación, tiene un papel importante en el tratamiento de los TIIC. En el caso del ECF la evidencia recomienda su uso, en especial la fibra soluble, ya que mejora el volumen de las heces y acelera el tránsito intestinal, y se emite con cautela la recomendación para la fibra insoluble por los efectos secundarios. En el caso del SII la evidencia es menor y mucho más heterogénea, y si bien mejora la consistencia y frecuencia de las evacuaciones, su efecto sobre el dolor abdominal (el síntoma que define al SII) es incierto. Por lo tanto, son necesarios más estudios de calidad para poder realizar recomendaciones específicas y conocer con claridad la dosis, tipo y fuente de fibra requerida, para el manejo de los síntomas en ECF, SII y otros TIIC. Por otra parte, el consumo de alimentos naturales ricos en fibra y la coadministración de fibras (p. ej., psyllium más inulina) son opciones prometedoras que deben de explorarse.

\section{FINANCIAMIENTO}

No se recibió ningún financiamiento.

\section{CONFLICTO DE INTERESES}

El Dr. José María Remes-Troche es miembro del comité asesor de Takeda, Alfasigma, Biocodex y Asofarma. Ha recibido honorarios como ponente por parte de Takeda, Chinoin, Ferrer y Alfasigma. La Dra. Karla Rocío García-Zermeño no tiene conflictos de interés.

\section{RESPONSABILIDADES ÉTICAS}

Protección de personas y animales. Los autores declaran que para esta investigación no se han realizado experimentos en seres humanos ni en animales.

Confidencialidad de los datos. Los autores declaran que han seguido los protocolos de su centro de trabajo sobre la publicación de datos de pacientes.

\section{Derecho a la privacidad y consentimiento} informado. Los autores declaran que en este artículo no aparecen datos de pacientes.

\section{BIBLIOGRAFÍA}

1. Lacy BE, Mearin F, Chang L, Chey WD, Lembo AJ, Simren M, et al. Bowel disorders. Gastroenterology. 2016:150(6):1393-407.

2. Gill SK, Rossi M, Bajka B, Whelan K. Dietary fibre in gastrointestinal health and disease. Nat Rev Gastroenterol Hepatol. 2021;18(2):101-16.

3. Zielinski G, Devries J, Shaw Craig SA, Bridges AR. Dietary Fiber Methods in Codex Alimentarius: current status and ongoing discussions. Cereal Foods World. 2013;58:148-52.

4. Food and Agriculture Organization/World Health Organization Codex Alimentarius commission. Codex Alimentarius: Guidelines on Nutrition Labelling CAC/GL 2-1985. Food and Agriculture Organization; 2010.

5. Deehan EC, Duar RM, Armet AM, Perez-Muñoz ME, Jin M, Walter J. Modulation of the gastrointestinal microbiome with nondigestible fermentable carbohydrates to improve human health. Microbiol Spectr. 2017;5(5).

6. Lovegrove A, Edwards CH, De Noni I, Patel H, El SN, Grassby T, et al. Role of polysaccharides in food, digestion, and health. Crit Rev Food Sci Nutr. 2017;57(2):237-53.

7. Cummings JH, Stephen AM. Carbohydrate terminology and classification. Eur J Clin Nutr. 2007;61(Suppl 1):S5-S18.

8. Food and Agriculture Organization. Food energy-methods of analysis and conversion factors. Report of a Technical Workshop no. 77. Roma: Food and Agriculture Organization; 2003.

9. Renard CMGC, Crepeau MJ, Thibault JF. Influence of ionic strength, pH and dielectric constant on hydration properties of native and modified fibres from sugar- beet and wheat bran. Ind Crop Prod. 1994;3:75-84.

10. Fleury N, Lahaye M. Chemical and physicochemical characterisation of fibres from Laminaria digitata (kombu breton): a physiological approach. J Sci Food Agric. 1991;55(3):389-400.

11. Ratanpaul V, Williams BA, Black JL, Gidley MJ. Review: Effects of fibre, grain starch digestion rate and the ileal brake on voluntary feed intake in pigs. Animal. 2019;13:2745-54.

12. Dikeman CL, Fahey GC. Viscosity as related to dietary fiber: a review. Crit Rev Food Sci Nutr. 2006;46:649-63.

13. Gawkowska D, Cybulska J, Zdunek A. Structure related gelling of pectins and linking with other natural compounds: a review. Polymers. 2018;10:76. 
14. Schweizer TF, Edwards CA, editores. Dietary fibre - A component of food. Springer; 1992. pp. 41-56.

15. Müller M, Canfora EE, Blaak EE. Gastrointestinal transit time, glucose homeostasis and metabolic health: Modulation by dietary fibers. Nutrients. 2018;10(3):275.

16. Chutkan R, Fahey G, Wright WL, McRorie J. Viscous versus nonviscous soluble fiber supplements: mechanisms and evidence for fiber-specific health benefits. J Am Acad Nurse Pract. 2012;24(8):476-87.

17. Vuksan V, Jenkins AL, Rogovik AL, Fairgrieve CD, Jovanovski E, Leiter LA. Viscosity rather than quantity of dietary fibre predicts cholesterol-lowering effect in healthy individuals. Br J Nutr. 2011;106(9):1349-52.

18. Topping DL, Oakenfull D, Trimble RP, Illman RJ. A viscous fibre (methylcellulose) lowers blood glucose and plasma triacylglycerols and increases liver glycogen independently of volatile fatty acid production in the rat. $\mathrm{Br}$ J Nutr. 1988;59(1):21-30

19. Anderson JW, Allgood LD, Lawrence A, Altringer LA, Jerdack GR, Hengehold DA, et al. Cholesterol-lowering effects of psyllium intake adjunctive to diet therapy in men and women with hypercholesterolemia: meta-analysis of 8 controlled trials. Am J Clin Nutr. 2000;71(2):472-9.

20. Bergmann JF, Chassany O, Petit A, Triki R, Caulin C, Segrestaa JM. Correlation between echographic gastric emptying and appetite: influence of psyllium. Gut. 1992;33(8):1042-3.

21. Dhital S, Gidley MJ, Warren FJ. Inhibition of $\alpha$ - amylase activity by cellulose: kinetic analysis and nutritional implications. Carbohydr Polym. 2015;123:305-12.

22. Qi J, Li Y, Yokoyama W, Masamba KG, Zhong F, Ma J. Cellulosic fraction of rice bran fibre alters the conformation and inhibits the activity of porcine pancreatic lipase. J Funct Foods. 2015;19:39-48.

23. Leng-Peschlow E. Interference of dietary fibres with gastrointestinal enzymes in vitro. Digestion. 1989;44:200-10.

24. Cummings JH, Macfarlane GT. The control and consequences of bacterial fermentation in the human colon. J Appl Bacteriol. 1991;70:443-59.

25. Cummings JH, Macfarlane GT, Englyst HN. Prebiotic digestion and fermentation. Am J Clin Nutr. 2001;73(2 Suppl):415S-20S

26. Todesco T, Rao AV, Bosello O, Jenkins DJ. Propionate lowers blood glucose and alters lipid metabolism in healthy subjects. Am J Clin Nutr. 1991;54:860-5.

27. Topping DL, Clifton PM. Short-chain fatty acids and human colonic function: roles of resistant starch and nonstarch polysaccharides. Physiol Rev. 2001;81:1031-64.

28. Roediger WE. Utilization of nutrients by isolated epithelial cells of the rat colon. Gastroenterology. 1982;83:424-9.

29. Flamm G, Glinsmann W, Kritchevsky D, Prosky L, Roberfroid M. Inulin and oligofructose as dietary fiber: a review of the evidence. Crit Rev Food Sci Nutr. 2001;41:35-62.

30. McRorie JW, Daggy BP, Morel JG, et al. psyllium is superior to docusate sodium for treatment of chronic constipation. Aliment Pharmacol Ther 1998;12:491- 7.

31. Davies GJ, Crowder M, Reid B, Diersing PS, Miner PB, Robinson M. Bowel function measurements of individuals with different eating patterns. Gut. 1986;27:164-9.

32. McRorie J, Greenwood-Van Meerveld B, Rudolph C. Characterization of propagating contractions in proximal colon of ambulatory mini pigs. Dig Dis Sci. 199;43:957-63.

33. Burkitt DP, Walker AR, Painter NS. Effect of dietary fibre on stools and the transit-times, and its role in the causation of disease. Lancet. 1972;2:1408-12.

34. Tomlin J, Read NW. Laxative properties of indigestible plastic particles. BMJ. 1988;297:1175-6.

35. Lewis SJ, Heaton KW. Roughage revisited: the effect on intestinal function of inert plastic particles of different sizes and shape. Dig Dis Sci. 1999;44:744-8.

36. Stephen AM, Cummings JH. Mechanism of action of dietary fibre in the human colon. Nature. 1980;284:283-4.

37. Camilleri M. Management of the irritable bowel syndrome. Gastroenterology. 2001;120:652-68
38. Camilleri M, Heading RC, Thompson WG. Clinical perspectives, mechanisms, diagnosis and management of irritable bowel syndrome. Aliment Pharmacol Ther. 2002;16:1407-30.

39. Hamer HM, Jonkers D, Venema K, Vanhoutvin S, Troost FJ, Brummer RJ. Review article: the role of butyrate on colonic function. Aliment Pharmacol Ther. 2008;27:104-19.

40. Gonlachanvit S, Coleski R, Owyang C, Hasler WI. Inhibitory actions of a high fibre diet on intestinal gas transit in healthy volunteers. Gut. 2004;53:1577-82.

41. McRorie J, Pepple S, Rudolph C. Effects of fiber laxatives and calcium docusate on regional water content and viscosity of digesta in the large intestine of the pig. Dig Dis Sci. 1998;43:738-45.

42. Marlett JA, Fischer MH. The active fraction of psyllium seed husk. Proc Nutr Soc. 2003;62:207-9.

43. Rigaud D, Paycha F, Meulemans A, Merrouche M, Mignon M. Effect of psyllium on gastric emptying, hunger feeling and food intake in normal volunteers: a double blind study. Eur J Clin Nutr. 1998;52:239-45.

44. Bianchi M, Capurso L. Effects of guar gum, ispaghula and microcrystallin cellulose on abdominal symptoms, gastric emptying, orocaecal transit time and gas production in healthy volunteers. Dig Liver Dis. 2002;34(Suppl 2):S129-33.

45. Frost GS, Brynes AE, Dhillo WS, Bloom SR, McBurney MI. The effects of fiber enrichment of pasta and fat content on gastric emptying, GLP-1, glucose, and insulin responses to a meal. Eur J Clin Nutr. 2003;57:293-8.

46. Kawasaki N, Suzuki Y, Urashima M, Nakayoshi T, Tsuboi K, Tanishima Y, et al. Effect of gelatinization on gastric emptying and absorption. Hepatogastroenterology. 2008;55:1843-5.

47. Grimes DS, Goddard J. Gastric emptying of whole meal and white bread Gut. 1977; 18:725-9.

48. Meyer JH, Gu Y, Elashoff J, Reedy T, Dressman J, Amidon G. Effects of viscosity and fluid outflow on postcibal gastric emptying of solids. Am J Physiol. 1986;250:G161-4

49. Russell J, Bass P. Canine gastric emptying of fiber meals: influence of meal viscosity and antroduodenal motility. Am J Physiol. 1985;249:G662-7.

50. Vincent R, Roberts A, Frier M, Perkins AC, MacDonald IA, Spiller RC. Effect of bran particle size on gastric emptying and small bowel transit in humans: a scintigraphic study. Gut. 1995;37:216-9.

51. Scott KP, Duncan SH, Flint HJ. Dietary fibre and the gut microbiota. Nutr Bull. 2008;33:201-11.

52. Duncan SH, Louis $\mathrm{P}$, Thomson JM, Flint HJ. The role of $\mathrm{pH}$ in determining the species composition of the human colonic microbiota. Environ Microbiol. 2009;11(8):2112-22.

53. Bouhnik Y, Flourie B, Riottot M, Bisetti N, Gailing MF, Guibert A, et al. Effects of fructo-oligosaccharides ingestion on fecal bifidobacteria and selected metabolic indexes of colon carcinogenesis in healthy humans. Nutr Cancer. 1996;2:21-9.

54. Slavin JL. Position of the American Dietetic Association: health implications of dietary fiber. J Am Diet Assoc. 2008;108:1716-31.

55. Barquera S, Rivera-Dommarco J, Campos I, Espinoza J, Monterrubio E. Consumo de fibra y sobrepeso en mujeres mexicanas en edad adulta. Nutrición Clínica. 2002;5(4):206-12.

56. Trowell H. Definition of dietary fiber and hypotheses that it is a protective factor in certain diseases. Am J Clin Nutr. 1976;29:417-27.

57. Burkitt DP, Meisner P. How to manage constipation with high-fiber diet. Geriatrics. 1979;34:33-5.

58. Remes-Troche JM, Coss-Adame E, Lopéz-Colombo A, Amieva-Balmori M, Carmona Sánchez R, Charúa Guindic L, et al. The Mexican consensus on chronic constipation. Rev Gastroenterol Mex (Eng Ed). 2018;83(2):168-89.

59. Carmona-Sánchez R, Icaza-Chávez ME, Bielsa-Fernández MV, GómezEscudero O, Bosques-Padilla F, Coss-Adame E, et al. The Mexican consensus on irritable bowel syndrome. Rev Gastroenterol Mex. 2016;81(3): 149-67.

60. Vasant DH, Paine PA, Black CJ, Houghton LA, Everitt HA, Corsetti M, et al. British Society of Gastroenterology guidelines on the management of irritable bowel syndrome. Gut. 2021;70(7):1214-40. 
61. Brusciano L, Gualtieri G, Gambardella C, Del Genio G, Tolone S, Lucido FS, et al. ESNM Guidelines on functional constipation in adults: Further reflections and considerations. Neurogastroenterol Motil. 2020;32(9):e13915.

62. Lacy BE, Pimentel M, Brenner DM, Chey WD, Keefer LA, Long MD, et al. ACG Clinical Guideline: Management of Irritable Bowel Syndrome. Am J Gastroenterol. 2021;116(1):17-44.

63. Bharucha AE, Pemberton JH, Locke GR. American Gastroenterological Association technical review on constipation. Gastroenterology. 2013;144: 218-38.

64. Mearín F, Ciriza C, Mínguez M, Rey E, Mascort JJ, Peña E, et al.; en nombre de la SEPD, la semFYC, la SEMERGEN y la SEMG. Guía de práctica clínica del síndrome del intestino irritable con estreñimiento y estreñimiento funcional en adultos: tratamiento (Parte 2 de 2). Aten Primaria. 2017;49(3):177-94

65. Guías Mundiales de la Organización Mundial de Gastroenterología. Estreñimiento: una perspectiva mundial [Internet]. Organización Mundial de Gastroenterología; noviembre de 2010 [consultado: marzo 2021]. Disponible en: https://www.worldgastroenterology.org/guidelines/global-guidelines/constipation/constipation-spanish

66. Guías Mundiales de la Organización Mundial de Gastroenterología. Síndrome de intestino irritable: una perspectiva mundial [Internet]. Organización Mundial de Gastroenterología; noviembre de 2010 [consultado: marzo 2021]. Disponible en: https://www.worldgastroenterology.org/guidelines/global-guidelines/irritable-bowel-syndrome-ibs/irritable-bowel-syndrome-ibs-spanish

67. Irritable bowel syndrome in adults: diagnosis and management. Clinical guideline [CG61] [Internet]. National Institute for Health and Care Excellence; 23 febrero 2008 [última actualización: 4 abril 2017]. Disponible en: www.nice.org.uk/guidance/cg61

68. Constipation in children and young people: diagnosis and management. Clinical guideline [CG99] [Internet]. National Instiotute for Health and Care Excellence; 26 mayo 2010 [última actualización: 23 febrero 2008]. Disponible en: https://www.nice.org.uk/guidance/cg99

69. Schmulson WM, Francisconi C, Olden K, Paíz LA, Bustos-Fernández L, Cohen $\mathrm{H}$, et al. Consenso Latinoamericano de Estreñimiento Crónico. Gastroenterol Hepatol. 2008;31(2):59-74.

70. Attaluri A, Donahoe R, Valestin J, Brown K, Rao SSC. Randomised clinical trial: dried plums (prunes) vs. psyllium for constipation. Aliment Pharmacol Ther. 2011;33:822-8.

71. Chan AOO, Leung G, Tong T, Wong NYH. Increasing dietary fiber intake in terms of kiwifruit improves constipation in Chinese patients. World J Gastroenterol. 2007;13:4771-5.

72. Chey SW, Chey WD, Jackson K, Eswaran S. Exploratory comparative effectiveness trial of green kiwifruit, psyllium, or prunes in US patients with chronic constipation. Am J Gastroenterol. 2021;116(6):1304-12.

73. Coss-Adame E, Garcia-Cedillo MF, Martinez HE, Delgado MKB, Bustillo AGR, Cassis NL. Randomized double blind controlled trial to assess the efficacy of agave fructans (tequilana weber blue variety) versus psyllium plantago in patients with chronic functional constipation. Gastroenterology. 2019;156(6):S-594.

74. Rao SSC, Yu S, Fedewa A. Systematic review: dietary fibre and FODMAPrestricted diet in the management of constipation and irritable bowel syndrome. Aliment Pharmacol Ther. 2015;41:1256-70.

75. Yang J, Wang HP, Zhou L, Xu CF. Effect of dietary fiber on constipation: A meta-analysis. World J Gastroenterol. 2012;8:7378-83.

76. Suares NC, Ford AC. Systematic review: the effects of fibre in the management of chronic idiopathic constipation. Aliment Pharmacol Ther. 2011;33:895-901.

77. Fenn GC, Wilkinson PD, Lee CE, Akbar FA. A general practice study of the efficacy of Regulan in functional constipation. Br J Clin Pract. 1986;40:192-7.

78. Ashraf W, Park F, Lof J, Quigley EM. Effects of psyllium therapy on stool characteristics, colon transit and anorectal function in chronic idiopathic constipation. Aliment Pharmacol Ther. 1995;9:639-47.
79. Nunes F, Nunes C, Levis E. A double-blind trial of a celandin, aloe vera and psyllium laxative preparation in adult patients with constipation. Rev Bras Med. 2005;62:35-7.

80. Lopez Roman J, Martinez Gonzalvez A, Luque A. Efecto de la ingesta de un preparado lácteo con fibra dietética sobre el estreñimiento crónico primario idiopático. Nutr Hosp. 2008;23:12-9.

81. Badiali D, Corazziari E, Habib FI, Tomei E, Bausano G, Magrini P, et al Effect of wheat bran in treatment of chronic nonorganic constipation. A double-blind controlled trial. Dig Dis Sci. 1995;40:349-56.

82. Hongisto SM, Paajanen L, Saxelin M, Korpela R. A combination of fibre-rich rye bread and yoghurt containing Lactobacillus GG improves bowel function in women with self-reported constipation. Eur J Clin Nutr. 2006;60:319-24

83. Christodoulides S, Dimidi E, Fragkos KC, Farmer AD, Whelan K, Scott SM. Systematic review with meta-analysis: effect of fibre supplementation on chronic idiopathic constipation in adults. Aliment Pharmacol Ther 2016;44:103-16

84. De Vries J, Le Bourgot C, Calame W, Respondek F. Effects of $\beta$-fructans fiber on bowel function: a systematic review and meta-analysis. Nutrients. 2019;11(1).

85. Mertz H, Naliboff B, Mayer E. Physiology of refractory chronic constipation. Am J Gastroenterol. 1999;94:609-15.

86. Voderholzer WA, Schatke W, Mühldorfer BE, Klauser AG, Birkner B, Müller-Lissner SA. Clinical response to dietary fiber treatment of chronic constipation. Am J Gastroenterol. 1997;92:95-8.

87. Eswaran S, Muir J, Chey WD. Fiber and functional gastrointestinal disorders. Am J Gastroenterol. 2013;108:718-27.

88. Shin JE, Jung HK, Lee TH, Jo Y, Lee H, Song KH, et al. Guidelines for the diagnosis and treatment of chronic functional constipation in Korea, 2015 revised edition. J Neurogastroenterol. 2016;22:383.

89. Design of Treatment Trials Committee, Irvine EJ, Whitehead WE, Chey WD Matsueda K, Shaw M, Talley NJ, et al. Design of treatment trials for functional gastrointestinal disorders. Gastroenterology. 2006;130:1538-51.

90. Manning AP, Heaton KW, Harvey RF. Wheat fibre and irritable bowel A controlled trial. Lancet. 1977;2:417-8.

91. Prior A, Whorwell PJ. Double blind study of ispaghula in irritable bowel syndrome. Gut. 1987;28:1510-3.

92. Lambert JP, Brunt PW, Mowat NA, Khin CC, Lai CK, Morrison V, et al. The value of prescribed 'high-fibre' diets for the treatment of the irritable bowel syndrome. Eur J Clin Nutr. 1991;45:601-9.

93. Rao SS, Yu S, Fedewa A. Systematic review: dietary fibre and FOD$\mathrm{MAP}$ - restricted diet in the management of constipation and irritable bowel syndrome. Aliment Pharmacol Ther. 2015;41:1256-70.

94. Nagarajan N, Morden A, Bischof D, King EA, Kosztowski M, Wick EC, et al. The role of fiber supplementation in the treatment of irritable bowel syndrome: a systematic review and meta-analysis. Eur J Gastroenterol Hepatol. 2015;27(9):1002-10.

95. Ford AC, Talley NJ, Spiegel BM, Fox-Orenstein AE, Schiller L, Quigley EM, et al. Effect of fibre, antispasmodics, and peppermint oil in the treatment of irritable bowel syndrome: systematic review and meta-analysis. BMJ. 2008;337:a2313.

96. Moayyedi P, Quigley EM, Lacy BE, Lembo AJ, Saito YA, Schiller LR, at al. The effect of fiber supplementation on irritable bowel syndrome: a systematic review and meta-analysis. Am J Gastroenterol. 2014;109(9):1367-74.

97. Remes-Troche JM, Taboada-Liceaga H, Gill S, Amieva-Balmori M, Rossi M, Hernández-Ramírez G, et al. Nopal fiber (Opuntia ficus-indica) improves symptoms in irritable bowel syndrome in the short term: a randomized controlled trial. Neurogastroenterol Motil. 2021;33(2):e13986.

98. López-Romero P, Pichardo-Ontiveros E, Avila-Nava A, Vázquez-Manjarrez N, Tovar AR, Pedraza-Chaverri J, et al. The effect of nopal (Opuntia ficus indica) on postprandial blood glucose, incretins, and antioxidant activity in Mexican patients with type 2 diabetes after consumption of two different composition breakfasts. J Acad Nutr Diet. 2014;114:1811-8. 